\title{
EL COMITÉ OLIMPICO INTERNACIONAL: ¿UN NOMBRE PARA LA ETERNIDAD?
}

\author{
Jeroni Sureda \\ Licenciado en Ciencias Politicas (itinerario internacional) \\ Universitat Autònoma de Barcelona
}

Resumen

Aunque el Comité Olimpico Internacional (COI) es tuna ONG especializada en la organización de los Juegos Olimpicos, su influencia en el sistema internacional ha ido en aumento desde su fundación en 1894 . Su impulsor, el Barón de Coubertin, incluyó dos objetivos de claro significado politico: la promoción de la amistad entre los pueblos del mundo mediante la práctica del deporte, $y$ la intención de garantizar una tregua olimpica para evitar que hubiera guerras durante la celebración de los Juegos. Con tales propósitos se establecio el COI como un organismo independiente de los gobiernos, pero necesitado de la colaboración de éstos.

A pesar de las criticas al COI por no cumplir sus objetivos iniciales, se le ha reconocido su papel politico en el escenario internacional. Después de la Primera Guerra Mundial las actividades del COl, y entre ellas los fuegos, permitieron que los estados las utilizasen para obtener reconocimiento o prestigio en la comunidad internacional.

Durante la guerra fría el COI sirvió para rebajar o, al menos, para dirimir las tensiones entre los dos bloques. En cualquier caso el COI, aun siendo una $O N G$, ha servido para reforzar el papel de los estados como actores principales del sistema.

Abstract

Although the International Olympic Committee is a INGO specialized on organising the $O$ lympic Games, it has had an increasing infuence in the international system ever since its foundation in 1894 . Its founder Baron de Coubertin included among its objectives swo which had a very obvious political significance. One was the strengthening of friendship amongst people from all over the world through the practice of sport, and another was the pledge to end all

1. Esce artículo tiene su origen en la memoria de licenciatura presentada por el autor en la Facultad de Ciencias Políticas y de Sociología en 1989. La investigación posterior sobre el rema fue posible gracias a la concesión, por parte de la Universitat Aurònoma de Barcelona, de una ayuda para un proyecro sobre "Jocs Olímpics i Relacions Internacionals", presentado por Esther Barbé i Jeroni Sureda al programa de "Grups pre-compecitius o nous projectes d'investigación. 
wars by means of an olympic truce which would take place during the celebration of the Games. With those objectives in mind the $1 O C$ was set up as an international body independent of governments, but requiring their goodwill.

The $10 C$ has been subject to criticism for it may not bave accomplished its originary goals, but its political role in the international arena has been manifest. After the frst world war the activities of the IOC and especially the Olympic Games showed the states how sport could be used as a way to foster international recognition or prestige. The end of the colonial empires after the second world war continued this tendency. In the years of the cold war the IOC helped ease tensions between the two blocks. In all cases, including the present international situation, the IOC, though a NGO itself, has nevertheless reinforced the role of the state as the main actor of the international system.

Desde su fundación en 1894, la historia del Comité Olímpico Internacional ha dado un ejemplo poco corriente de supervivencia. Prototipo de iniciativa privada y expresión del cosmopolitismo de las clases dominantes en la Europa de la "Belle Epoque", nació en el marco de la "paz armada", y de un sistema europeo de equilibrio multipolar. Después de las dos guerras mundiales el Comité se tuvo que adaptar a las nuevas circunstancias: los desequilibiros de entreguerras, primero, y el equilibrio bipolar dominado por el temor a la bomba atómica, después. En todos los casos el COI no solamente consiguió superar los sucesivos retos ante los cambios internacionales, sinó que además vio aumentar su influencia en la comunidad internacional.

Los Juegos de Barcelona, celebrados dentro de una situación mundial de búsqueda de un nuevo orden internacional, pusieron de manifiesto la importancia del COI y del movimiento olímpico en general al conseguir que representantes de ciento setenta y dos países participaran en el acontecimiento. Los retos continúan. Víctima de su éxito, el olimpismo se enfrenta a los peligros de su propio crecimiento.

Sin embargo, peor suerte ha tenido el COI a la hora de ser considerado como actor en el sistema internacional. Las causas son de carácter teórico, pero también académico.

\section{EL COI, ¿ACTOR INTERNACIONAL?}

Desde el punto de vista teórico, el paradigma realista únicamente admitía al estado como actor internacional, basándose en los conceptos de soberania, reconocimiento y control de un territorio. Los temas incluidos en la agenda realista: seguridad, lucha por el poder, disuasión etc., tampoco tenían mucho que ver con un fenómeno como el olímpico, cuyos dirigentes además se es- 
forzaban una y otra vez en rechazar cualquier implicación del olimpismo con la política.

Los cambios económicos y políticos que ocurrieron después de la Segunda Guerra Mundial: descolonización, bloques militares, crisis energética, etc., obligaron a los teóricos de las relaciones internacionales a hablar de pluralidad de actores y a romar en cuenta la inclusión de actores no-estatales, que si bien no eran nuevos, no habían sido considerados como tales.

A partir de los criterios exigidos por los paradigmas que admiten una pluralidad de actores en relaciones internacionales, y que han sido recogidos por García en este mismo número, podemos preguntarnos si el COI responde a dichos criterios. Para responder a la pregunta hace falta un estudio de su estructura, su autonomía respecto a los estados, su capacidad de mover recursos $y$, finalmente, su grado de influencia en el sistema internacional (Hocking $\& \mathrm{x}$ Smith, 1990:75).

Otra causa de la poca atención prestada al COI por parte de los teóricos de las relaciones internacionales hay que buscarla en la falta de tradición académica al respecto (Kyrolaynen \& Varis, 1981:54). Si bien se ha ido aceptando el papel como actores de algunos organismos supraestatales o de las empresas transnacionales, peor suerte han tenido los actores subestatales o algunas organizaciones no gubernamentales, y entre ellas el Comité Olímpico Internacional. Esta poca consideración por cierto tipo de actores "menores" podía deberse al deseo de la comunidad científica por no perder prestigio y también a una traducción al campo de la teoría de las tendencias políticas de los investigadores (Hietanen \& Varis, 1982:75; Kanin, 1978:526).

Veamos pues si el COI es merecedor de esta falta de atención teórica y, en caso negativo, cuáles son las funciones que ha desempeñado y desempeña en el sistema internacional.

\section{LA ESTRUCTURA DEL COI}

El Comité Olímpico Internacional es el órgano rector del Movimiento Olímpico y, en consecuencia, de los Juegos Olímpicos. Los demás componentes del Movimiento son las Federaciones Internacionales (FI) de los deportes, que compiten en los Juegos, y los Comités Olímpicos Nacionales (CON).

Las Federaciones Internacionales están encargadas de la organización técnica de las competiciones y el establecimiento de reglas por las cuales estos deportes se rigen. Los Comités Olimpicos Nacionales, por su parte, son comités establecidos en un territorio, generalmentc un estado, y cuya misión principal es asegurar la representación de su país en los Juegos Olímpicos. El reconocimiento de un Comité Nacional está supeditado al previo reconocimiento de 
un cierto número de federaciones de aquel país por parte de las correspondientes federaciones internacionales. En teoría son organismos independientes de los gobiernos del territorio que representan.

La posibilidad de incluir al Comité Olímpico Internacional entre los actores del sistema internacional no viene solamente dada, como se afirma a menudo, por el hipotético valor del deporte como factor de distensión internacional. Su vinculación con el mundo de las relaciones internacionales es más estrecha. La misma decisión del COI sobre la conveniencia o no de admitir un comité nacional en el seno del olimpismo, le hace tener un papel político. El acuerdo del COI de diciembre de 1992, según el cual únicamente serán reconocidos los comités olímpicos nacionales de estados admitidos en la ONU, puede evitar en el futuro algunos problemas, pero vincula aún más el olimpismo a la situación política y no excluye situaciones difíciles a largo plazo. Recordemos que en los años cincuenta y sesenta el COI se negó a reconocer el Comité de Taiwan como representante de toda la China, al contrario de lo que ocurría en el sistema central de las Naciones Unidas, donde el representante del gobierno de Taipeh ocupaba el sillón que habria correspondido a la República Popular que controlaba la China Continental.

La estructura del COI no ha variado sustancialmente desde su constitución en 1894. El Comité está formado por un número no fijo de miembros vitalicios, en la actualidad son noventa y uno, elegidos por el sistema de co-optación, es decir por votación directa de los que ya forman parte del mismo. Los miembros lo son a título individual y, en teoría, no representan a su país de origen.

La composición del primer Comité es un buen reflejo del carácter masculino, elitista y eurocéntrico del COI: de sus quince miembros, ninguna mujer entre ellos, cinco tenian títulos nobiliarios, al menos dos eran generales, y todos ellos tenían orígenes europeos, aunque uno era argentino y otro era de Nueva Zelanda. Cien años después se ha roto la exclusividad masculina, blanca y elitista, pero no su predominio.

Las decisiones del Comité son ejecutadas por una comisión ejecutiva compuesta por el presidente del Comité, un cargo elegido por ocho años y renovable cada cuatro, y por otros ocho miembros tambien elegidos.

La composición del COI se asemeja más a la de un club privado, en el que predominan hombres blancos con títulos nobiliarios, que no a la de un organismo internacional. Su sistema de elección es igual al de otras organizaciones nacidas en el siglo XIX, como la Cruz Roja. No han faltado propuestas para la transferencia de sus funciones a la UNESCO (discurso del Presidente Kekkonen de Finlandia, el 30-10-1971 en Jyväskylä), o la de convertir el comité en una asamblea democrática con un miembro por cada comité olímpico nacional (Alexandriakis \& Krotee, 1988:328; Brohm, 1981:24). 
Sin embargo incluso quienes admiten la dificultad en legitimar un sistema electivo tan poco democrático reconocen en éste ciertas ventajas, como el alto grado de cohesión interna, cohesión hecha todavía más evidente por el carácter secreto de sus deliberaciones, evitando así que salgan a la luz o se filtren al exterior las disensiones en el seno del Comité (Nafzinger, 1971:190; Ivanov, 1982:80; Collomb, 1988:242). La no existencia del derecho de voto ha impedido también que se produjeran situaciones como las del sistema central de las Naciones Unidas.

\section{LOS ORIGENES DEL COI: LOS OBIETIVOS DE COUBERTIN}

Los orígenes del movimiento olímpico hay que buscarlos en la convergencia de varios factores: la investigación arqueológica en la antigua Olimpia, impulsada por alemanes, la tradición del deporte amateur favorecido por algunos miembros de la aristocracia británica, el espíritu regeneracionista de una parte de la sociedad francesa dispuesta a superar la derrota de $1870 \mathrm{y}$, sobre todo, la visión de Pierre de Frédy, barón de Coubertin (Mandell, 1986:84).

El congreso para el restablecimiento de los Juegos Olímpicos, celebrado en la Sorbona en 1894 estableció las reglas del olimpismo, de las que se conservan la mayoría: celebración de Juegos cada cuatro años, cambio de sede olímpica en cada ocasión, y elección de miembros del recién creado Comité Olímpico por el sistema de co-optación (Merce Varela, 1992: 64).

Los objetivos de la restauración olímpica expresados por Coubertin iban más allá de la práctica deporriva. Dos de ellos eran claramente políticos: uno era la voluntad de hacer de la práctica deportiva y de las competiciones olímpicas un medio para el acercamiento de las personas de clases diferentes y de pueblos de países, razas y religiones diferentes.

El otro objetivo era más concreto: at recuperar la tradición de los Juegos Olímpicos de la Antigiiedad, las competiciones olímpicas deberían contribuir al fin de las guerras gracias a la "tregta sagrada" que se respectaria durante la celebración de las pruebas de la restaurada olimpíada.

Según Coubertin, el éxito del ideal olímpico exigía la independencia de los poderes políticos y de ahí la necesidad de contar con miembros de clases influyentes. Al mismo tiempo, esta independencia requería la colaboración con los estados.

Coubertin tuvo el valor de proclamarse, a través del COI, depositario de la tradición olímpica clásica y a partir de entonces luchó con éxito, primero para imponer sus reglas para la celebración de los Juegos Olímpicos de la Nueva Era y, después, para convertir al COI en el órgano rector del deporte olímpico a escala mundial. 
Detrás de la iniciativa olímpica, ahora centenaria, había una visión liberal, en el sentido que la palabra tenía en Europa en aquel tiempo, de las posibilidades de la iniciativa privada sin la intervención del estado, pero también una suptesta falta de conciencia de los cambios sociales que se iban produciendo a finales del siglo XIX. El espíritu olímpico, a pesar de abogar por una mayor camaradería entre los pueblos de la Tierra, ponía el énfasis en la importancia del esfuerzo individual, en un momento en el que las clases trabajadoras veían el asociacionismo como el único camino para avanzar hacia mejoras sociales.

Las críticas al olimpismo suelen centrarse en el no cumplimiento de los ideales olímpicos expuestos por el fundador (Brohm, 1981:50), sin embargo, estos principios no estuvieron exentos de contradicciones:

1. Elitismo. La llamada a la participación olímpica de jóvenes de todas las clases sociates y todos los paises suponía que solamente los que dispusieran de suficientes fondos económicos podrían tomarla en consideración, al estar prohibida en aquel entonces la presencia de deportistas profesionales. Para favorecer la participación había dos caminos: o rebajar las exigencias sobre el amateurismo o dejar que los estados subvencionasen a los deportistas. En ambos casos ello suponía romper las aspiraciones del COI de conservar el amateurismo y el apoliticismo (Mandell, 84:196). Si se prohibía el profesionalismo, la práctica del deporte quedaba limitada a las clases que no tuvieran que trabajar.

2. El eurocentrismo. El COI no planteaba cambios en la estructura del sistema internacional, en aquel momento de carácter eurocéntrico. El ideal de ruptura de barrcras de estados y razas era limitado. Había participantes de países americanos, de Sudáfrica, Nueva Zelanda y Japón, pero, exceptuando el caso de los japoneses, los demás participantes de otros continentes eran también de origen europeo. Otro ejemplo del eurocentrismo lo tenemos en el tipo de deportes olímpicos, todos ellos europeos, y no precisamente por respeto a los Juegos de la Antiguedad. Cien años después y salvo excepciones, como el judo o el taekwondo, continúa la preponderancia de los deportes de origen aristocrático y europeo (Rose,1986:185). El eurocentrismo del COI, al igual que el del paradigma realista, ha sido criticado como desfasado (Dougherty \& Pfaltzgraff, 1990: 124). Sin embargo, queda abierto el debate sobre el mantenimiento del eurocentrismo en el sistema internacional actual a pesar del desplazamiento de las áreas de poder a otras regiones del planeta.

3. La desmilitarización del deporte. La contradicción de este postulado no es producto tanto del elevado número de militares entre los deportistas 
y entre los miembros de los órganos rectores del olimpismo, sino a las constantes llamadas de Coubertin a hacer del deporte un medio para crear jóvenes "viriles" y "patriotas". Así, algunos críticos han visto en el olimpismo una especie de religión laica con un culto al superhombre (Brohm, 1981:48).

4. El supuesto apoliticismo. El Comité se empeñó desde el principio en destacar su carácter apolítico y su independencia de los gobiernos, pero no pudo dejar de lado la importancia de la política. El propio barón había estudiado en el Instituto de Estudios Políticos de París y sabia que hacía falta la ayuda de los poderes públicos para el progreso del olimpismo. No deja de ser significativo que los primeros Juegos Olímpicos ya tuvieron consecuencias políticas: la dimisión del primer ministro de Grecia, por su oposición a los gastos olímpicos (Ohl, 1977:75).

Cuando el sistema dentro del cual habia nacido el COI desapareció con el estalido de la Gran Guerra de 1914, podía parecer que el movimiento olímpico se hundiría con él. No en vano los Juegos Olímpicos previstos para Berlin en 1916, no solamente no consiguieron una "tregua olímpica" según la cual se pararían las hostilidades entre los contendientes, sino que además los Juegos ni tan siquiera se celebraron. La suspensión de los Juegos de 1916, igual que ocurriría con los de 1940 y 1944 , demostraba que si bien el olimpismo no podía acabar con las guerras, en cambio los Juegos no se podían desarrollar si no había paz. De esta manera el COI quedaba obligado a luchar por la paz mundial, aunque fuera únicamente para defender sus intereses.

\section{DEL COSMOPOLITISMO AL INTERNACIONALISMO}

La situación internacional surgida después de la Primera Guerra Mundial supuso el final del equilibrio anterior, de las monarquías centroeuropeas y del cosmopolitismo elitista, que habían servido de marco al COI. También supuso la ruptura de la unidad ideológica del sistema con el nacimiento de la Unión Soviética. Aún así, y sin variar su estructura, el COI supo adaptarse a las nuevas circunstancias: masificación de la práctica deportiva y nacimiento del deporte como espectáculo de masas, afianzamiento del ritual olímpico y la internacionalización de los Juegos Olímpicos mediante el incremento del interés de los estados europeos por las competiciones deportivas olimpicas.

1. Masificación del deporte. En el período de entreguerras, el deporte se populariza y masifica, y la selección de los mejores deportistas se hace dentro de un ámbito de población relarivamente más amplio. El ejército en Europa 
y las universidades en los Estados Unidos sirvieron para reclutar deportistas olímpicos que, de esta manera, podrían dedicarse a practicar deporte. Esta ampliación de la base social curiosamente ayudó a reforzar la importancia del COI y de los Juegos.

2. Ritual Olímpico. Coubertin había querido convertir el olimpismo en una especie de religión laica que hiciese del deporte un culto a la superación individual, a la amistad y al cuerpo humano. Se puede poner en duda si estos objetivos han llegado a cumplirse, pero en cualquier caso la popularización de los Juegos creó los ritos que hoy forman parte del olimpismo, entre ellos el alojamiento de los deportistas en una villa olímpica ad hoc, que apareció por primera vez en los Juegos de Los Angeles de 1932.

Los ritos olímpicos no sirvieron para crear el tipo de religión propugnado por Coubertin, pero, en cambio, sirvieron para reforzar el sentimiento de cohesión social y de identificación con un grupo, cumpliendo así algunas de las funciones que, según Durkheim, siempre habian tenido todas las religiones. Sin embargo, en este caso el grupo no sería una clase dirigente, ni los practicantes de un deporre determinado, sino los miembros de un estado.

3. Inter-nacionalización. Si en un primer momento se pudo mantener la ficción de que los Juegos eran competiciones entre individuos de diferentes países, unos Juegos inter-personales, después de la Primera Guerra Mundial el intervencionismo de los estados en el deporte hizo que las competiciones olímpicas fuesen cada vez más competiciones inter-nacionales, o, mejor aún, inter-estatales. La estatalización de los Juegos se hizo evidente mediante el uso de toda la parafernalia de himnos, banderas, desfiles de participantes agrupados por estados y, muy especialmente, mediante la clasificación, si bien no de una manera oficial, de los países según el número de medallas que sus atletas conseguían.

Ahora bien, la intervención de los estados en el deporte fue posible gracias a otros indicios no tan evidentes como los anteriores, como el incremento de los deportes en equipo y la necesidad de mejor preparación de los atletas ante la competencia de los participantes de otros países. Todo eilo requería una infraestructura de tal magnitud que facilitaba la intromisión de los gobiernos de los estados en el proceso formativo de los deportes, en una operación que ahora se consideraba susceptible de producir dividendos políticos.

El ejemplo más claro de participación del estado en unos Juegos Olímpicos lo tenemos en el tantas veces comentado caso de la olimpiada de Berlin en 1936. Aquella celebración olímpica ha sido presentada como una lamentable excepción en la historia olímpica. Sin embargo, la explotación de unos Juegos para hacer propaganda de un régimen y la implicación de toda la administra- 
El Comité Olímpico Internacional: ¿un nombre para la eternidad?

ción estatal para conseguir su buen funcionamiento, que fueron en su momento dos rasgos criticados, han sido imitados a partir de entonces por los organizadores de los Juegos posteriores.

4. Amenazas al monopolio del COI. En ese período de entreguerras hubo intentos de romper el monopolio del COI por parte de asociaciones deportivas internacionales dependientes de la Internacional Socialista y de la Comunista, mediante la organización de competiciones paralelas. La afrenta suponía, paradójicamente, un reconocimiento hacia los valores del deporte, considerado hasta entonces como expresión burguesa.

La competencia entre comunistas y socialistas quiró fuerza al olimpismo obrero, y cuando finalmente, gracias a la política de frentepopulismo, las dos corrientes se unieron, el único estado socialista, la Unión Soviética, ya había entrado en la Sociedad de Naciones, y no estaba interesada en enfrentarse al olimpismo oficial. Si bien no hay presencia de deportistas soviéticos en unos Jutegos Olímpicos hasta 1952, el COI salió reforzado, tras el periodo de entreguerras, en su papel de foro capaz de poner a prueba el prestigio de los estados.

\section{LA MAYORIA DE EDAD: EL COI EN EL SISTEMA INTERNACIONAL BIPOLAR}

Los cambios producidos después de la Segunda Guerra Mundial, que llevaron a un sistema bipolar y al nacimiento de los estados independientes del Tercer Mundo no hicieron más que aumentar la importancia del COl y, detrás de él, de todo el movimiento olímpico. Todo ello sin modificar formalmente las estructuras originarias del Comité.

Esta tercera etapa olímpica supuso la entrada de cuatro rasgos característicos que se han conservado en los años noventa: crecimiento del Movimiento Olímpico, aumento de las actividades del COI, cambios en su financiación y una universalización del Movimiento (Guttmann, 1984).

1. Crecimiento del Movimiento Olímpico. El crecimiento del movimiento olímpico desde 1945 ha llegado a tal grado, que el gigantismo actual se considera un peligro a corto plazo, ya que puede hacer desaparecer los Juegos. Este crecimiento es producto de diferentes factores y se traduce en:

a) Un aumento en el número de miembros del COl.

b) Un aumento en el número de comités olímpicos nacionales.

c) Una mayor participación de las mujeres, las cuales en un principio estaban ausentes casi por completo de los Juegos. 
d) Un aumento en el número de deportes participantes en los Juegos Olímpicos y, por consiguiente, en las cifras de deportistas olímpicos.

2. Incremento de las Actividades. El COI aumentó su influencia no solamente a causa del crecimiento del movimiento olímpico, sino también a causa de otro tipo de competiciones de carácter regional en la organización de las cuales también cstuvo presente. Ejemplos de estas compericiones serían los Juegos del Mediterráneo o los Juegos Asiáticos. Y, en el plano de la cooperación internacional, el COI puso en marcha un plan de ayuda al deporte en el Tercer Mundo mediante su programa de "Solidaridad Olímpica", hecho en cooperación con la UNESCO.

Esta cooperación representa el reconocimiento del COI por parte de la comunidad internacional como la entidad deportiva global por antonomasia, como el gobierno mundial del deporte, en un momento en el cual el interés de la población del mundo por el deporte no deja de subir.

El gigantismo del movimiento olímpico supuso un nuevo reto. La multiplicación de comités olímpicos nacionales y su organización en una asamblea puso en peligro la hegemonía del COI dentro del movimiento olímpico $y$, sin embargo, éste supo mantenerse. La estructura de los comités olímpicos nacionales, en muchos casos con vínculos gubernamentales, ha permitido al COI convertirse en la cúpula de un entramado mixto - privado y gubernamental-, elemento esencial para su enraizamiento en el sistema.

3. Cambios en la financiación. Hasta el año 1948 los ingresos del COI se basaban principalmente en la cotización de sus miembros. A partir de aquel momento se decidió que las ciudades organizadoras pagaran una cantidad para el mantenimiento del olimpismo. Con la entrada de la televisión en los estadios, los ingresos del COI se dispararon.

Este cambio suponía un reforzamiento de la autonomía financiera del COI, pero además evitaba las presiones a las cuales estaban sometidas algunas organizaciones internacionales, como la misma UNESCO e incluso la ONU, las cuales funcionan gracias a las cuotas de los estados miembros. Sin embargo, los nuevos ingresos no dejaban de representar un nuevo peligro, puesto que implicaban el establecimiento de vínculos con las compañias de televisión y abrían paso a la comercialización de los Juegos y, según algunos críticos, a su control por parte de empresas transnacionales (Simson \& Jennings, 1992). Este pretendido poder de empresas transnacionales sobre el movimiento olímpico no ha sido, sin embargo, analizado. ¿Ejercen las empresas su influencia en el sistema internacional a través de organismos como et $\mathrm{COI}$ ? ¿o tienen objetivos crematísticos? Dada la diversidad de intereses de las diferentes compañías, la segunda opción parece más creáble. En cualquier 
El Comiré Olímpico Internacional: ¿un nombre para la eternidad?

caso, el aumento del poder económico del COI refuerza su papel como actor internacional.

4. Universalización del Movimiento Olímpico. En el año 1951 se produjo la entrada de la URSS en el olimpismo con el reconocimiento del Comité Olímpico Soviético. Con ello se acababa el monolirismo ideológico del COI mantenido desde su creación, pero al mismo tiempo consolidaba su posición y le dejaba sin competencia posible por parte de organizaciones obreras, tal como había ocurrido en el período anterior. Asi el COI dejaba de ser percibido como un enemigo de la clase obrera.

En un momento de guerra fría, la inclusión de miembros de países del Este en el COI fue vista desde Occidente como una adulteración de la trayectoria olímpica. La participación de la URSS en los Juegos de Helsinki de 1952 fue considerada como una muestra de la entrada de la política en el olimpismo: "La política mostró una vez más su cara fea en el movimiento olímpico" (Graham \& Ueberhorst, 1976: 188).

Sin embargo, las reuniones del COI y la celebración de los Juegos sirvieron como marco de encuentro entre los dos bloques. Si este papel fue positivo o no depende de la postura que el investigador tenga sobre el papel de la guerra fría en sí. ¿Sirvió el olimpismo de lugar de confrontación? ¿ Fue una multinacional del deporte defendiendo los intereses comerciales de sus patrocinadores $y$, en consecuencia, un instrumento del capitalismo? Hechos como la presencia de los atletas de las dos Alemanias bajo una sola bandera, son algunos ejemplos, si bien limitados, del uso de los Juegos como factor de distensión (Milza, 1988:267).

La caida del muro de Berlín en el año 1989 y el fin de la división del mundo en dos bloques hacía prever que los Juegos de 1992 serían los de la distensión. Otros cambios regionales, como el proceso político de África Austral, permitieron la participación de Namibia y de Sudáfrica en Barcelona, en unos Juegos en los que, por primera vez después de muchos años, no hubo boicots. Además, los conflictos internacionales no impidieron que bajo la propia bandera o arropados bajo el estandarte olímpico hubiera representantes de prácticamente todos los países del mundo. Pero el desfile de Barcelona evidenciaba la realidad mundial cambiante: la multitud de banderas que sustitúan la extinta URSS suponían un interrogante para la futura configuración del este europeo.

Los cambios mundiales pusieron a prueba una vez más la estructura del COI. Igual que había ocurrido en 1919 y en 1945, varios de los miembros del Comité habían sido elegidos en función de un historial que era incompatible ahora con la nueva situación de los países a los cuales pertenecían. Pero la ficción según la cual los miembros del COI no representan a sus estados de ori- 
"Papers": Revista de Sociologia

gen, permitió que altos oficiales soviéticos continuaran en su puesto, como antes aristócratas zaristas lo habian hecho en 1919.

A pesar de los cambios, el COI demostró en Barcelona que su poder de convocatoria, que su influencia, no había disminuido. La situación de guerra civil en los Balcanes no fue obstáculo para que representantes de todas las partes en conflicto pudieran participar en los Juegos. EI hecho que el llamamiento a la paz en la antigua Yugoslavia no fuera escuchado también ponia en guardia sobre los límites de la influencia del COI y del movimiento olímpico en la escena internacional.

\section{EL PAPEL DEL COI EN EL ACTUAL SISTEMA INTERNACIONAL}

Según la terminología oficial empleada por los dirigentes olímpicos, el $\mathrm{COI}$, y detrás de él todo el movimiento olímpico, son elementos que favorecen el acercamiento entre los pueblos y la distensión mundial. La base para tales afirmaciones radica en el papel positivo que se da al deporte olímpico, al favorecer los contactos entre gentes de culturas diferentes, la aceptación de unas reglas y una cierta homogeneización cultural (Mc Intosh 1984:80). Los idolos olímpicos, desde Paavo Nurmi a Vitali Scherbo, dejan de ser considerados como representantes de un país concreto y se convierten en patrimonio de todos (Gritti,1975:153).

El deporte olímpico, al igual que la música, sirve para avanzar hacia la creación de una cultura compartida en todo el mundo, gracias sobre todo al efecto amplificador de los medios de comunicación, pero ello no significa que esta influencia pueda trasladarse al campo de las relaciones internacionales. El aumento de los intercambios culturales o económicos no supone necesariamente un aumento en el nivel de integración o distensión. Por otra parte, el elemento competicivo que es inherente a cualquier práctica deportiva ha hecho creer a otros que el deporte fomenta la agresividad humana (Goldstein, 1983).

Ninguna de las dos hipótesis, sobre el valor intrínsecamente positivo o agresivo del deporte, ha podido ser validada en el campo de las relaciones internacionales.

El que existan posturas tan dispares sobre el valor del deporte se debe a su carácter polivalente y neutro. El deporte por sí mismo no sería ni bueno ni malo, sino que podría ser usado para objetivos diversos (Gueldenpfenning, 1985:203). Según Stolyarov, un sociólogo ruso, el deporte solamente puede tener un valor positivo si los deportistas y los espectadores han asumido un sistema de valores capaz de apreciarlo (Stolyarov-Sanadze, 1984:38).

$\mathrm{Si}$ admitimos que el COl es algo más que un organismo dependiente de la voluntad de un estado o de un bloque de estados y que además tiene un 
cierto grado de autonomía, podremos pensar que existe la posibilidad teórica de influencia en el sistema internacional por parte del Comité. Más difícil es establecer la forma, el sentido, que pueda tener esta influencia.

La supervivencia del COI parece demostrar que, a través de los Juegos Olímpicos, sirve para fomentar la cohesión de los estados. Estudios como los de Vayrinen o Kropke apuntan a las Olimpiadas como factores que crean o refuerzan el sentimiento de grupo (Kropke, 1974: 25; Vayrinen, 1982:126). El grado de cohesión obtenida dependerá de la existencia de otros factores. Si tomamos como ejemplo las medallas conferidas a los vencedores en las compericiones olímpicas, la misma medalla no hará el mismo servicio si se da al deportista de un estado o al de ozro.

En segundo lugar, los Juegos Olímpicos sirven para facilitar la entrada a la comunidad internacional a aquellos estados que tienen problemas de reconocimiento internacional.

Finalmente, los éxitos de los atletas en unos Juegos, o el éxito de su organización, suponen respectivamente un aumento del prestigio del estado al cual pertenecen estos atletas o de la ciudad organizadora.

Ninguno de los tres factores mencionados supone, en principio, una aportación a la distensión o a la comprensión entre los estados. Porque si bien se ha probado el papel integrador de los Juegos y de la práctica deportiva en general en el seno de una sociedad concreta, este hecho no permite sacar la conclusión automática según la cual este papel sea aplicable ał campo de las relaciones internacionales. Incluso hay autores que creen que la cohesión de un grupo, o que el sentimiento de unidad dentro de un estado, lo que hacen es fomentar la agresividad en contra de otros grupos o estados.

Lo mismo se podría decir de los otros dos factores. Si un estado tiene problemas de reconocimiento diplomático o está «mal visto" por la comunidad internacional, puede ser a causa de haber roto algunas normas impuestas por la comunidad. En este sentido su prestigio, vía deportiva, podria ser considerado como peligroso para la estabilidad del sistema internacional.

El movimiento olímpico ayuda pues a reforzar el papel de los estados como actores principales en eí sistema internacional vigente. Si hacemos una interpretación realista como la de Hedley Bull, en el sentido que el orden internacional sirve de marco para rebajar las tensiones, entonces los Juegos Olímpicos, al reforzar un orden internacional asentado sobre los estados, sirven para facilitar la distensión.

La afirmación anterior supone una toma de postura en el debate interparadigmático y, en este sentido, son muchas las críticas que se hacen al COl y a los Juegos por esta función de apoyo al papel de los estados.

Muchos creen que la existencia de los estados es uno de los motivos principales de conflictos en el mundo, y por ello el olimpismo debería reforzar el 
papel de los pueblos y no de los entes estatales y, por ello, la participación de los atletas debería hacerse a título individual, con lo cual desaparecería una gran parte del ritual olímpico: banderas, himnos, equipos seleccionados por los comités olímpicos nacionales, etc. También debería haber una sede permanente de los Juegos, se propone Atenas (Seurin, 1980:13).

De llevarse a cabo las propuestas anteriores, seguramente desaparecerian muchos de los vínculos entre olimpismo y estados, pero también la posibilidad del COl para jugar un papel relevante en el sistema internacional.

Puede octrrir que haya grupos étnicos - actores subestatales- que no se encuentren identificados con el estado bajo cuya bandera se ven obligados a participar. Ahora bien, las quejas de estos grupos no van tanto hacia la supresión de los estados sino a su sustitución. La historia del olimpismo nos muestra que, si bien se ha producido utilización política de los Juegos, tanto por parte de los gobiernos como por parte de otros grupos, esta utilización solamente ha tenido éxito cuando se ha empleado la única atma admitida en la lucha olímpica: la de la participación deportiva. Un ejemplo paradigmático en este sentido, lo encontramos en el caso de Finlandia, que se sirvió de sus éxitos olímpicos para consolidar el nuevo estado. En cambio no se ha probado la obtención de resultados cuando se han urilizado las olimpíadas con fines políticos con otras armas que no fueran las deportivas. ¿Hubo alguna rentabilidad política en los hechos de Munich de 1972, o en los boicors de 1976, 1980 y 1984 ?

La garantía de supervivencia del COI y de los Juegos Olímpicos viene dada por las propias limitaciones del Comité. Al ser un ente especializado, sus decisiones pueden tener repercusiones políticas, pero en la mayoría de los casos son de tipo técnico y deportivo, y por ello no representan un peligro para los estados.

Así pues, la respuesta a la pregunta de si el olimpismo puede jugar un papel, y mejor aún un papel positivo, en el mundo de las relaciones internacionales dependerá de la voluntad de otros actores del sistema internacional y, muy especialmente, de los estados (Kanin, 1978:516). Un ejemplo de esta limitación la dio el presidente Carter al justificar el boicot de los Juegos de Moscú cuando dijo "creo en la conveniencia de mantener la política gubernamental fuera del olimpismo, pero están en juego intereses superioresn ("deeper issues are at stake») (Dpt. Of State Bulletin, March 1980: 51).

"Quien mucho abarca poco aprieta" asegura un dicho popular. La creciente intervención del estado en todos los ámbitos ha facilitado paradójicamente la posibilidad de permeabilidad de sus estructuras por parte de otros actores. Por eso el COI, a pesar de su composición aparentemente desfasada, tiene garantizada su continuidad, porque ayuda a mantener el papel de los estados en el sistema. De esta manera la promoción de los ideales olímpicos es una buena inversión estatal. 
El Comité Olímpico Internacional: ¿un nombre para la eternidad?

\section{BIBLIOGRAFIA}

Brohm, J. (1981), Le Mythe Olympique, Paris, Christian Bourgois Editeur.

Collomb, P. (1988), Sport, Droit et Relations Internationales, Patís, Economica.

Dougherty, J. y Pfaltzgraff, R. (1990), Contending Theories of International Relations, New York, Harper-Collins.

Goldstein, J. (1983), Sports Violence, New Yotk, Springet.

Graham, P. y Ueberhorst, H.(1976), The Modern Olympics. West Point (N.X.), Leisute Press.

Gritti, J.(1975), Sport a la Une, Paris, Armand Colin.

Gueldenpfenning, S. (1985), "Sport in the Peace Movement-A Challenge for the Sport Science", International Review for the Sociology of Sport, num. 20/3, pp. 203-208.

Gutmann, Allen (1984), The Games Must Go on. Avery Brundage and the Olympic Movement. New York, Columbia University Press.

Hietanen, A. y Varist, T. (1982), "Sport and International Understanding: A Survey of the Strucrure and Trends of International Sports Cooperation", Current Research on P'iece and Violence, núm. 2-3, pp. 75-107.

Hocking, B y Smith, M. (1990), World Politics. An Introduction to International Politics, New York, Harvester-Wheatsheaf.

Ivanov, A. (1982), "On the Olympic Charrer of the International Olympic Committee", en Solakov, A (ed.) Topical Problems of the Olympic Movement, Sofia, Sofia Press.

Kanin, D. (1978), "The Olympic System: Transnational Sport Organization and the Politics of Cultural Exchange», en Lowe B. \& Kanin D. (eds.), Sport and International Relations, Champaign, (IIl.), Sriper.

Krpopke, R. (1974), "International Sports and the Social Sciences", Quest, Spring Issue, June, pp. 25-31.

Luschen, G. (1984), "Sport, International Conflict and Conflict Resolution", en Ilmarinen, M. (ed.), Sport and International Understanding. Proceedings of the Congress about Sport and International Understanding-Helsinki 1982, Berlin-Heidelberg, Springer-Verlag GmbH \& Co.

Mandell, R. (1986), Historia Cultural del Deporte, Barcelona, Ed. Bellaterra.

McInrosh, P. (1984), «Sistcms of Value and International Sport», en Olin K. (ed.), Contribution of Sociology to the Study of Sport, Jyväskylä, Jyväskylan Yliopiston Monistuskeskus.

Mercè Varela, A. (1992), Pierre de Coubertin, Barcelona, Edicions 62-Caixa de Catalunya.

Milza, P. (1988), "Sport et Politique: Les Enjeux de Séoul, Politique International, eté núm. 3, p. 262.

Nafzinger, J.A. (1971), "The Regulation of Transnational Sports Competition: Down from Mount Olympus", Vanderbilt Journal of Transnational Law, vol.5, núm 1.

Ohl, P.E. (1977), La Guerre Olympique, Paris, Robert Laffont. 
"Papers": Revista de Sociologia

Rose, D. (1986), "Theoretical and Practical Confusion about the Olympic Games", en Redmond, G. (ed.) Sport and Politics, Champaign, (Ill.), Human Kinetics. Seuxin, B. (1980), "The Future of the Olympic Games and Tomorrow's Sporc", F.I.E.P. Bulletin, vol. 50, núm. 2. p. 7.

Simson, V. y Jennings, A. (1992), Los Señores de los Anillos, Barcelona, El Triangle. Stoliarov, V. y Sanadze, L. (1984), "The Role of International Sporting Ties in Strengthening Peacc and Understanding between Nationsm, cn Ilmarinen, M. (ed.), Sport and International Understanding (vide supra).

Vayrinen, R. (1982), "Nationalism and Internationalism in Sports", Current Research on Piece and Violence, núm. 2-3, pp. 123-130. 